\title{
Integration of Interactive CFD Simulations with AR and VR for Educational Use in CRE
}

\author{
Serkan Solmaz, ${ }^{\mathrm{a}^{*}}$ Tom Van Gerven ${ }^{\mathrm{a}}$ \\ ${ }^{a}$ Department of Chemical Engineering, KU Leuven, Leuven 3001, Belgium \\ serkan.solmaz@kuleuven.be
}

\begin{abstract}
Engineering simulations have since long been considered an imperative design and analysis tool for chemical reaction engineering (CRE). Notably, reactor and process design are domains in which multiphysics computational fluid dynamics (CFD) simulations are applied to understand phenomena that are difficult, time-consuming or expensive to be explored with experiments. On the other hand, advanced post-processing methods and virtual reality can enable high-quality educational content with engineering simulations. Nevertheless, the utilization of these tools in education is still underdeveloped. This study explores the potential of integration of multiphysics CFD simulations with AR/VR and its educational use. By iterating on this potential we present a generic system architecture with inter-disciplinary consideration of two building stones: (1) Recent advancements in CFD \& multiphysics simulations, and (2) Emerging immersive technologies. A case study is demonstrated applying the generic methodology in order to illustrate the integration. The study promotes a rich and engaging environment with engineering simulations where interactivity can be empowered to entertain learners with AR/VR. This will primarily assist enthusiasts to develop feasible complementary environments with simulations.
\end{abstract}

Keywords: Chemical reaction engineering, Computational fluid dynamics, Augmented reality, System architecture, Learning environment.

\section{Introduction}

Multiphysics CFD simulations, one of the most applied computer-assisted process engineering (CAPE) tools, have been a maturing method increasing comprehension of engineering design and analysis. However, the educational implementation of this tool is still directly made with engineering software which has a primitive educational context, thereby preventing students to understand challenging concepts. Augmented reality (AR) and virtual reality (VR) have been getting prevalent in society today including research and education (Suh and Prophet, 2018). The integration of interactive CFD simulations in $\mathrm{AR} / \mathrm{VR}$ reveals supportive features such as advanced visualizations, interactivity, attractiveness of digital media and so forth. In that sense, a virtual environment with CFD simulations could yield remedies for this primitive context.

The interest in CRE and CFD on multiple scales has recently convinced researchers to consider AR/VR by promoting virtual process engineering (Ge et al., 2019; Li, 2015). Despite this, several ambiguities are still present concerning the integration of CFD simulations with AR/VR in terms of system architecture. The term system architecture has come to be used as a portrayal of the system that consists of software and hardware including their functionalities and interactions (Blach et al., 1998). The present study develops a methodology for a sustainable system architecture to integrate the desired digital simulation contents into AR/VR.

\subsection{Interactive Multiphysics $C F D$ Simulations in $A R / V R$}

Multiphysics CFD simulations for CRE have received much interest in the past decade. To date, several commercial and open-source software packages have been developed providing miscellaneous options to design and analyze reactive flow phenomena (Tian et al., 2018). These result in a broad database in the literature illustrating and solving multiple spatio-temporal cases. 
Integrating CFD simulations with AR/VR recently received scholarly attention due to technical complexities related to system architecture. Berger et al. was among the first ones to develop a plausible workflow for implementing CFD simulation results in AR/VR using the Unity3D game engine. However, these early studies exhibit a manual integration of CFD and AR/VR with dedicated instruments, with no consideration given to system architecture (Berger and Cristie, 2015). Various approaches have since then been reported to deal with the hurdles encountered. Kim et al. proposed a cloud computing network to entertain users' interaction in VR with CFD simulations (Kim et al., 2018). More recently, Fukuda et al. examined system design parameters and network connections for visualization of CFD simulations at indoor environment with AR and mapped simulation results (Fukuda et al., 2019). Concerning data processing, Lin et al. developed a data format to reduce the data size of CFD dataset in a mobile device-based AR (Lin et al., 2019). A recent review by $\mathrm{Li}$ et. al on engineering simulations in AR found that systems were mostly developed for very unique elements due to the use of dedicated software, hardware, and platform. This eventually resulted in several constraints in terms of data- and workflow, thus the system architecture. It was also highlighted that most of the studies dismissed two-way coupling between CFD simulation and user-interaction, and merely focused on the visualization of simulation results ( $\mathrm{Li}$ et al., 2017). Moreover, low-accuracy real-time CFD simulations with the LatticeBoltzmann method were highlighted as an alternative to traditional CFD simulations. Harwood et al. claimed that interaction of real-time CFD simulations in VR generated with Unreal Engine 4 is applicable and can present a vivid advancement to simplify the system architecture. It was also mentioned that the integration of real-time CFD simulation with VR/AR brings its own hurdles for post-processing of CFD simulations (Harwood et al., 2018). Real-time CFD simulations are currently merely viable for oversimplified models, for instance, laminar external fluid flow. This raises many questions about whether real-time simulations could be applicable in the near future. An important downside of the above-mentioned studies is that they neglect a two-way coupling between CFD simulator and the end-user environment. Another major criticism is the need for dedicated software, hardware, data format, data processing method and thereby system architecture (Li et al., 2017). Every change or replacement of a building block in the system may affect the whole system architecture due to changes in the dataand workflow.

\subsection{Utilization of CFD Simulations in Education}

In spite of the friendly user-interfaces (UI), engineering simulation software is still lacking an educational approach. This can hence prevent lecturers to use these tools directly in education. Alternatively, a fully-integrated CFD simulation in VR might present a remedy to prevail over this uneasiness. Few studies have been published to support traditional learning methods with CFD simulations. Chemical process (Li, 2015), internal combustion engine (Tian and Abraham, 2014), biomedicine (Quam et al., 2015), and food engineering (Wong et al., 2010) were the recent application fields where CFD was promoted as a complementary asset. Glessmer et al. assessed the efficacy of lowaccuracy real-time CFD simulations to teach the basics of fluid mechanics. Results showed that both students' interest and comprehension on the topic were increased with using real-time CFD in an active learning environment (Glessmer and Janßen, 2017). The fundamental goal of the present research is to scrutinize the system design process of AV/VR with interactive multiphysics CFD simulations. Present study first proposes a methodology for a robust integration of CFD to AR/VR, then discusses a case study to illustrate the development of the digital environment, and finally dwells on the future direction of the proposed methodology.

\section{Methodology}

In our proposed methodology, integration of CFD simulations with AR/VR was demonstrated. To provide a sustainable integration, an approach was followed pinpointing each of the steps taken. This methodology presents a scaffold for a generic system architecture targeting two-way coupling. 


\subsection{Analysis of the system}

To begin with, exploratory studies on integrating CFD simulation with AR/VR were performed using the methods available in the literature as reported in the introduction. CFD simulations from conventional to real-time and virtual environments from VR to AR were reviewed and evaluated in terms of the components of the system. Once a complete one-way integration was achieved, such as the visualization of streamlines in AR, steps taken throughout the integration were noted in the workflow and clustered with regards to the relevance of components. In this way, system components in the methodology were inclusively determined. This exploratory study indicated that software, hardware, bridging, add-in, networking and internet of things are the fundamentally required building blocks to develop a two-way coupled system architecture for interactive CFD simulations in AR/VR.

In the second place, it was found that system architecture and end-user environment were also influenced by indirect components, namely academic content (Li et al., 2017) and learning analytics (Lai and Bower, 2019). Hamilton et al. reviewed an approach to design learning environments with relevant academic content and technological support. The study expressed the significant interference of indirect components to design a learning environment (Hamilton et al., 2016). In the present methodology only academic content was considered as an indirect component because of our focus on the system architecture.

\subsection{Proposed methodology}

A methodology was ultimately developed to establish a sustainable system architecture. A more generic, versatile integration was aimed at instead of using, for instance, a mere data format. The system architecture consists of components, requirements and indicators. Figure 1 outlines the proposed methodology integrating CFD simulation with AR/VR.

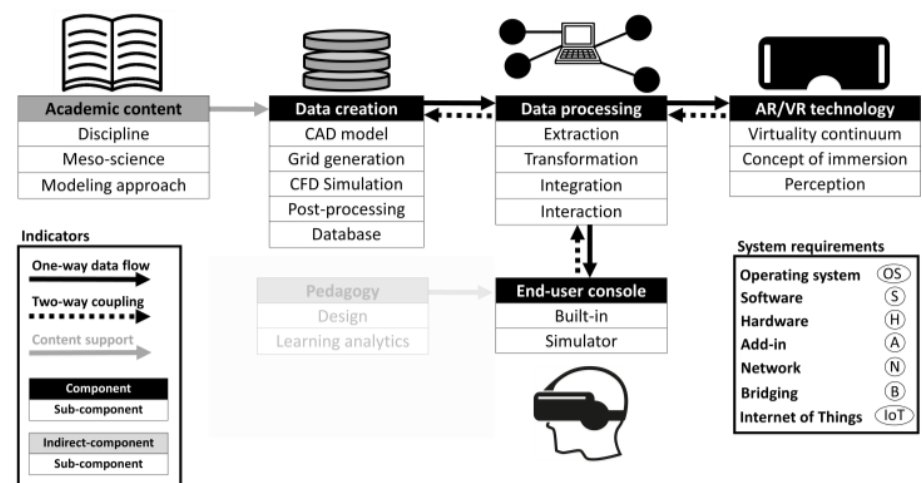

Figure 1. Proposed methodology for the system architecture to integrate process engineering simulation tools with AR/VR.

While components are the stages of workflow comprising dedicated tasks throughout the development of the end-user console, sub-components are the branches holding a responsibility to achieve devoted tasks. Indirect-components do not have any interference on the workflow, but may affect the requirements of components. System requirements are the specified platforms utilizing a variety of options to execute the tasks attained for each component. A sub-component must also be designed by selecting at least one of the requirements. Lastly, indicators are illustrated as binders of the components to outline the data flow. We believe this method represents a viable alternative to the literature.

\section{Case study}

A case study was carried out to evaluate the proposed system architecture. For simplicity, a benchmark CFD study was selected where we evaluated the run-time of calculations and validation. Being a mature application in CRE, the mixing process inside a stirred tank was simulated and results were visualized with mobile marker-based AR. Requirements used for each component were selected intuitively based on functionality, 
license, and customer support. All of the softwares used in the following sections were either open-source or accessible with a free personal-developer license. Neither commercial utilization or broadcasting was aimed at.

\subsection{CFD Simulations}

Initially, 3D geometry was designed with FreeCAD v0.18.4. Grid generation, preprocessing and calculations were performed through the use of pimpledymfoam in OpenFOAM v3.0.1. Extraction of the simulation dataset was done with ParaView v5.6.0. Once data processing was performed, the dataset was stored in the internal hard disk. The hardware employed was a Dell Precision 5530 Notebook (processor Intel(R) Core (TM) i7-8850H CPU @ 2.60GHz, 2592 MHz, 6 Cores, 12 Logical processors) with both Linux Ubuntu 16.04 and Microsoft Windows 10 as operating systems.

\subsection{Development of AR application}

Due to the customer support, Unity 3D 2019 game engine was chosen to develop the AR application. Although several AR software are available on the market, Vuforia was applied which provides a plug-in to enable its functionalities in Unity. Hence, by using Unity with the Vuforia plug-in, the required software for the development of AR was limited to one single platform. Interaction between the AR application and the real environment was realized with marker-based detection as an easy-to-adapt feature. Any kind of marker can be implemented depending on the developers' preferences. A procedure before final use should be set up online via the website of Vuforia to optimize detection and stability of the selected marker. In the present study human-computer interaction (HCI) was implemented by applying virtual buttons with Vuforia. This feature allowed to increase user-immersion further. The same laptop was used for the development of the application.

\subsection{Integration}

In Table 1 the workflow used in the case study is shown. Direct import of CFD data to Unity was the bottleneck because these software were using non-identical data formats. Therefore it was evident that an intermediate software must be considered to make processing possible. Blender v2.8 was chosen due to its versatile features for modeling 3D datasets. By comparing import and export data formats of ParaView, Blender and Unity, data processing without any data loss was only attainable with a single method. Once CFD simulation was completed and saved as raw $\mathrm{C}++$ document, the data was then processed with ParaView and extracted to '.x3d' format. Afterwards Blender was used to transform '. $\mathrm{x} 3 \mathrm{~d}$ ' to '.fbx', thereby making the dataset usable in Unity without any data loss. Not surprisingly, a major hindrance was found in the post-processing of the CFD results. Generating an iso-surface from the streamline visualized in Unity was impractical. Both iso-surfaces and streamlines should separately be post-processed in ParaView prior to data processing. Despite this handicap, ParaView and Blender are both environments that are based on Python programming language. A bridge can, therefore, be applicable between ParaView and Unity to solve this issue.

Table 1 . Workflow of the case study developed based on the proposed methodology.

\begin{tabular}{cccccccc}
\hline Component & \multicolumn{5}{c|}{ Data creation } & \multicolumn{2}{c}{ Data processing } \\
\hline $\begin{array}{c}\text { Side- } \\
\text { component }\end{array}$ & CAD & Grid & Simulation & $\begin{array}{c}\text { Post- } \\
\text { processing }\end{array}$ & Database & Extraction & Transformation \\
\hline OS & Windows & Ubuntu & Ubuntu & Ubuntu & $\begin{array}{c}\text { Ubuntu } \\
\text { File- }\end{array}$ & Windows & Windows \\
Software & FreeCAD & OpenFOAM & OpenFOAM & ParaView & $\begin{array}{c}\text { Pased } \\
\text { laptop }\end{array}$ & laptop & laptop \\
$\begin{array}{c}\text { Hadrware } \\
\text { Add-in }\end{array}$ & laptop & laptop & laptop & laptop & Plen \\
$\begin{array}{c}\text { Network } \\
\text { Bridging }\end{array}$ & Pyhton & C++ & C++ & Pyhton & & Pyhton & Pyhton \\
$\begin{array}{c}\text { IoT } \\
\text { Order of } \\
\text { workflow }\end{array}$ & 1 & 2 & 3 & 4 & 6 & 5 & 7 \\
\hline
\end{tabular}


Table 1. Workflow of the case study developed based on the proposed methodology.

\begin{tabular}{|c|c|c|c|c|c|c|}
\hline \multicolumn{2}{|c|}{ Data processing } & \multicolumn{3}{|c|}{ AR/VR technology } & \multicolumn{2}{|c|}{ End-user console } \\
\hline Integration & Interaction & $\begin{array}{c}\text { Virtuality } \\
\text { continuum }\end{array}$ & $\begin{array}{l}\text { Concept of } \\
\text { immersion }\end{array}$ & Perception & Built-in & Simulator \\
\hline Windows & Android & Windows & Android & Android & Windows & Android \\
\hline Unity & Vuforia & Unity & Vuforia & Vuforia & Unity & $\begin{array}{c}\text { Mobile } \\
\text { application }\end{array}$ \\
\hline laptop & smartphone & laptop & smartphone & smartphone & laptop & smartphone \\
\hline Vuforia & Marker & Vuforia & $\begin{array}{l}\text { Virtual } \\
\text { button }\end{array}$ & Camera & smartphone & Marker \\
\hline $\begin{array}{l}\text { LAN \& } \\
\text { WLAN }\end{array}$ & & & & & USB & \\
\hline $\mathrm{C \#}$ & $\mathrm{C \#}$ & $\mathrm{C} \#$ & $\mathrm{C} \#$ & $\mathrm{C \#}$ & $\mathrm{C} \#$ & $\mathrm{C \#}$ \\
\hline 11 & 12 & 8 & 9 & 10 & 13 & 14 \\
\hline
\end{tabular}

Applying system requirements for each sub-component gave a detailed workflow of the case study. It revealed that various softwares are available in the literature for tasks executed by each sub-component with different data formats. Nevertheless, direct integration was not possible without an intermediate data processing tool. A laptop and a smartphone were used to perform integration and to build the end-used application for mobile augmented reality, respectively. Interestingly, to give extra functions to both software and hardware, add-ins were found advantageous. This made workflow much more structured to express necessities clearly for each components. Another requirement that should not be dismissed was the network. It was experienced that the internet connection was needed to activate Vuforia plug-in inside Unity. The design of the marker for AR was also done through the website of Vuforia. To generate an AR application in a smartphone using Unity, a connection was realized between laptop and smartphone with a USB cable. The study also showed that communication between sub-components could be compatible with bridging different programming languages. Eventually, a two-way coupling might be developed between components. Further elaborations showed that the internet of things (IoT) could be an imperative asset to increase the level of communication and interaction among sub-components. The order of workflow presents a sequence for the particular case study. It can be readjusted in the other studies on purpose, for instance bridging.

\subsection{End-user Console}

A mobile application was generated with Unity 2019 to visualize the simulation results into mobile marker-based AR. Smartphone-based head-mounted display with Samsung A20e with Android Pie 9 was used to run the application for end-user interaction. Figure 2 illustrates the mobile AR application developed.
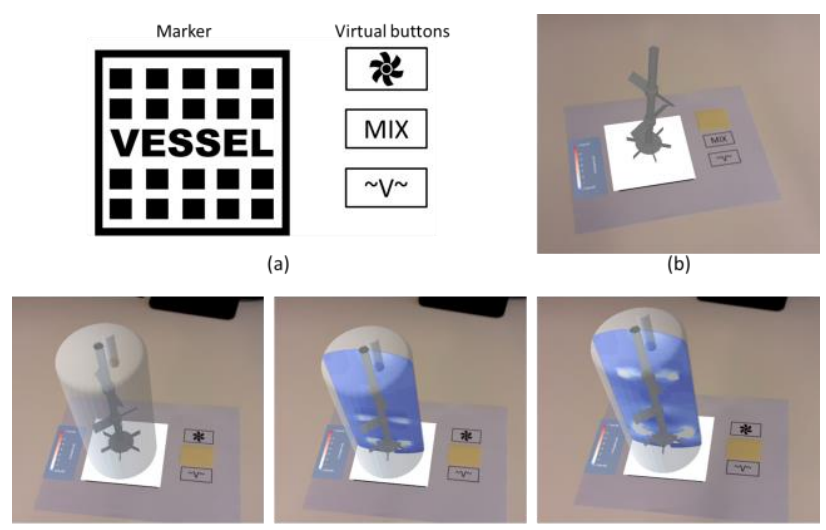

Figure 2. Mobile marker-based AR application; (a) marker and virtual buttons; (b) impeller; (c) visualization of transient CFD simulation. 


\section{Conclusion}

In this paper, we explored the integration of multiphysics CFD simulations with AR/VR. A methodology was presented to develop a sustainable system architecture. The results from this study indicate that the integration has significant potential to exploit multiphysics CFD simulation in AR/VR. This opens up new opportunities for developers dealing with engineering simulations to create desired digital content and integrate them with AR/VR. The research mainly stressed the importance of a sustainable system architecture by means of components, requirements, and indicators. A generic system architecture was proposed allowing alternative workflow and dataflow in the integration. Special attention should be paid by developers on dataflow to the integration of simulation data with AR/VR without the need of compromising on certain required datasets. This also underlines the importance of a database including a variety of complexities for CFD modeling from real-time to high accuracy. The present study only investigated a single way of dataflow. Therefore, future work will concentrate on accomplishing a two-way coupling. This will eventually deploy a sustainable integration.

\section{Acknowledgements}

This project has received funding from the European Union's EU Framework Programme for Research and Innovation Horizon 2020 under Grant Agreement 812716. This publication reflects only the author's view exempting the Community from any liability. Project website: https://charming-etn.eu/.

\section{References}

Berger, M., Cristie, V., 2015. CFD Post-processing in Unity3D. Procedia Computer Science 51, $2913-2922$. https://doi.org/10.1016/j.procs.2015.05.476

Blach, R., Landauer, J., Rösch, A., Simon, A., 1998. A highly flexible virtual reality system. Future Generation Computer Systems 14, 167-178. https://doi.org/10.1016/S0167-739X(98)00019-3

Fukuda, T., Yokoi, K., Yabuki, N., Motamedi, A., 2019. An indoor thermal environment design system for renovation using augmented reality. Journal of Computational Design and Engineering 6, 179-188.

Ge, W., Guo, L., Liu, X., Meng, F., Xu, J., Huang, W.L., Li, J., 2019. Mesoscience-based virtual process engineering. Computers \& Chemical Engineering 126, 68-82.

Glessmer, M., Janßen, C., 2017. Using an Interactive Lattice Boltzmann Solver in Fluid Mechanics Instruction. Computation 5, 35. https://doi.org/10.3390/computation5030035

Hamilton, E.R., Rosenberg, J.M., Akcaoglu, M., 2016. The Substitution Augmentation Modification Redefinition (SAMR) Model: a Critical Review and Suggestions for its Use. TechTrends 60, 433-441.

Harwood, A.R.G., Wenisch, P., Revell, A.J., 2018. A real-time modelling and simulation platform for virtual engineering design and analysis, in: Proceedings of 6th European Conference on Computational Mechanics (ECCM 6) and 7th European Conference on Computational FluidDynamics (ECFD 7). Glasgow, UK.

Kim, M., Yi, S., Jung, D., Park, S., Seo, D., 2018. Augmented-Reality Visualization of Aerodynamics Simulation in Sustainable Cloud Computing. Sustainability 10, 1362. https://doi.org/10.3390/su10051362

Lai, J.W.M., Bower, M., 2019. How is the use of technology in education evaluated? A systematic review. Computers \& Education 133, 27-42. https://doi.org/10.1016/j.compedu.2019.01.010

Li, J., 2015. Approaching virtual process engineering with exploring mesoscience. Chemical Engineering Journal 278, 541-555. https://doi.org/10.1016/j.cej.2014.10.005

Li, W., Nee, A., Ong, S., 2017. A State-of-the-Art Review of Augmented Reality in Engineering Analysis and Simulation. Multimodal Technologies and Interaction 1, 17. https://doi.org/10.3390/mti1030017

Lin, J.-R., Cao, J., Zhang, J.-P., van Treeck, C., Frisch, J., 2019. Visualization of indoor thermal environment on mobile devices based on augmented reality and computational fluid dynamics. Automation in Construction 103, 26-40. https://doi.org/10.1016/j.autcon.2019.02.007

Quam, D.J., Gundert, T.J., Ellwein, L., Larkee, C.E., Hayden, P., Migrino, R.Q., Otake, H., LaDisa, J.F., 2015. Immersive Visualization for Enhanced Computational Fluid Dynamics Analysis. Journal of Biomechanical Engineering 137, 031004. https://doi.org/10.1115/1.4029017

Suh, A., Prophet, J., 2018. The state of immersive technology research: A literature analysis. Computers in Human Behavior 86, 77-90. https://doi.org/10.1016/j.chb.2018.04.019

Tian, Y., Demirel, S.E., Hasan, M.M.F., Pistikopoulos, E.N., 2018. An overview of process systems engineering approaches for process intensification: State of the art. Chemical Engineering and Processing - Process Intensification 133, 160-210. https://doi.org/10.1016/j.cep.2018.07.014

Tian, Z.F., Abraham, J., 2014. Application of Computational Fluid Dynamics (CFD) in Teaching Internal Combustion Engines. International Journal of Mechanical Engineering Education 42, 73-83.

Wong, S.Y., Connelly, R.K., Hartel, R.W., 2010. Enhancing Student Learning in Food Engineering Using Computational Fluid Dynamics Simulations. Journal of Food Science Education 9, 90-97. 